\title{
Peakon, Solitary and Smooth Periodic Wave Solutions for a Modification of the $K(2,2)$ Equation
}

\author{
Qing Meng ${ }^{1}$ and Bin $\mathrm{He}^{2}$ \\ ${ }^{1}$ Department of Physics, Honghe University, Mengzi, Yunnan 661100, China \\ ${ }^{2}$ College of Mathematics, Honghe University, Mengzi, Yunnan 661100, China \\ Correspondence should be addressed to Bin He; hebinmtc@163.com
}

Received 30 October 2013; Revised 22 December 2013; Accepted 2 January 2014; Published 23 February 2014

Academic Editor: Turgut Öziş

Copyright (c) 2014 Q. Meng and B. He. This is an open access article distributed under the Creative Commons Attribution License, which permits unrestricted use, distribution, and reproduction in any medium, provided the original work is properly cited.

\begin{abstract}
We consider a modification of the $K(2,2)$ equation $u_{t}=2 u u_{x x x}+2 k u_{x} u_{x x}+2 u u_{x}$ using the bifurcation method of dynamical systems and the method of phase portraits analysis. From dynamic point of view, some peakons, solitary, and smooth periodic waves are found and their exact parametric representations are presented. Also, the coexistence of peakon and solitary wave solutions is investigated.
\end{abstract}

\section{Introduction}

To study the role of nonlinear dispersion in the formation of patterns in the liquid drop, Rosenau and Hyman [1] showed that, in a particular generalization of the KdV equation,

$$
u_{t}+\left(u^{m}\right)_{x}+\left(u^{n}\right)_{x x x}=0, \quad m>0,1<n \leq 3,
$$

which is called $K(m, n)$ equation. They obtained solitary wave solutions with compact support in it, which they called compactons. For the case $m=n$ ( $m$ is an integer), these compactons had the property that the width was independent of the amplitude. Rosenau [2] also studied the $K(m, n)$ equation:

$$
u_{t}+a\left(u^{m}\right)_{x}+\left(u^{n}\right)_{x x x}=0,
$$

where $a$ is a constant. He investigated nonlinear dispersion and compact structures [3], nonanalytic solitary waves [2], and a class of nonlinear dispersive-dissipative interactions [4]. In [5, 6], general solutions to the $K(n, n)$ equation were studied. In [7], the nonlinear equation $K(m, n)$ was studied for all possible values of $m$ and $n$. Tian and Yin investigated shock-peakon and shock-compacton solutions for $K(m, n)$ equation by variational iteration method in [8]. The $K(m, n)$ equation with generalized evolution and time-dependent damping and dispersion was investigated and the 1-soliton solution was obtained in [9]. Biswas considered the following $K(m, n)$ equation with generalized evolution term:

$$
\left(u^{l}\right)_{t}+a u^{m} u_{x}+b\left(u^{n}\right)_{x x x}=0,
$$

and presented a solitary wave ansatz and obtained a 1-soliton solution [10]. Some soliton solutions of (3) were obtained in $[11,12]$. In 2010, Ambrose and Wright [13] considered the following equation:

$$
u_{t}=2 u u_{x x x}-u_{x} u_{x x}+2 k u u_{x}
$$

which is called a modification of the $K(2,2)$ equation:

$$
u_{t}=2 u u_{x x x}+6 u_{x} u_{x x}+2 k u u_{x}
$$

where $k \neq 0$ is a constant. They demonstrated that, for this equation, there are compactly supported traveling wave solutions and the Cauchy problem possesses a weak solution which exists locally in time.

In this paper, we consider the following modification of the $K(2,2)$ equation using the bifurcation theory and the method of phase portraits analysis [14-17]:

$$
u_{t}=2 u u_{x x x}+2 k u_{x} u_{x x}+2 u u_{x},
$$

where $k$ is an integer. The bifurcations of different traveling waves in parameter space and the phase diagrams will be 
given. Some exact peakon, solitary, and smooth periodic wave solutions will be presented.

Let $\xi=x-c t$ where $c \neq 0$ is the wave speed. By using the travelling wave transformation $u(x, t)=\phi(\xi)$ we reduce (6) to the following ordinary differential equations:

$$
-c \phi^{\prime}=2 \phi \phi^{\prime \prime \prime}+2 k \phi^{\prime} \phi^{\prime \prime}+2 \phi \phi^{\prime} .
$$

Integrating (7) once with respect to $\xi$, we have

$$
g-c \phi=2 \phi \phi^{\prime \prime}+(k-1)\left(\phi^{\prime}\right)^{2}+\phi^{2}
$$

where $g$ is the integral constant.

Letting $y=d \phi / d \xi$, we get the following planar system:

$$
\frac{d \phi}{d \xi}=y, \quad \frac{d y}{d \xi}=\frac{g-c \phi-\phi^{2}-(k-1) y^{2}}{2 \phi} \text {. }
$$

System (9) is a three-parameter planar dynamical system depending on the parameter set $(k, c, g)$. Since the phase orbits defined by the vector field of system (9) determine all travelling wave solutions of (6), we should investigate the bifurcation sets and phase portraits of system $(9)$ in $(\phi, y)$ phase plane as the parameters $k, c$, and $g$ are changed.

We know that a peakon solution of (6) corresponds to a heteroclinic orbit of system (9) and a solitary wave solution of (6) corresponds to a homoclinic orbit of system (9). Similarly, a periodic orbit of system (9) corresponds to a periodic wave solution of (6). Thus, to investigate peakons, solitary, and periodic waves of (6), we should find all heteroclinic orbits, homoclinic orbits, and periodic annuli of system (9) depending on the parameter space of this system.

The rest of this paper is organized as follows. In Section 2, we discuss the bifurcation sets and phase portraits of system (9), where explicit parametric conditions will be derived. In Section 3, we give some exact peakon, solitary, and smooth periodic wave solutions of (6). A short conclusion will be given in Section 4.

\section{Bifurcation Sets and Phase Portraits of System (9)}

Using the transformation $d \xi=2 \phi d \tau$, it carries (9) into the Hamiltonian system:

$$
\frac{d \phi}{d \tau}=2 \phi y, \quad \frac{d y}{d \tau}=g-c \phi-\phi^{2}-(k-1) y^{2} .
$$

Since both systems (9) and (10) have the same following first integrals:

when $k \neq-1, k \neq 0$, and $k \neq 1$,

$$
\phi^{k-1}\left(y^{2}-\frac{g}{k-1}+\frac{c \phi}{k}+\frac{\phi^{2}}{k+1}\right)=h,
$$

when $k=-1$,

$$
\phi^{-2} y^{2}+\frac{g}{2 \phi^{2}}-\frac{c}{\phi}+\ln (\phi)=h,
$$

when $k=0$,

$$
\phi^{-1} y^{2}+\frac{g}{\phi}+\phi+c \ln (\phi)=h
$$

and when $k=1$,

$$
y^{2}+\frac{1}{2} \phi^{2}+c \phi-g \ln (\phi)=h,
$$

then the two systems above have the same topological phase portraits except the line $\phi=0$. Obviously, $\phi=0$ is an invariant straight-line solution of system (9).

Next, we focus on the cases $k \neq-1, k \neq 0$, and $k \neq 1$ in (6) without mentioning it further.

Write $\Delta_{1}=c^{2}+4 g, \Delta_{2}=g /(k-1)$. Clearly, when $\Delta_{1}>$ 0 , system (10) has two equilibrium points at $A_{1,2}\left(\phi_{1,2}, 0\right)$ in $\phi$-axis, where $\phi_{1,2}=\left(-c \pm \sqrt{\Delta_{1}}\right) / 2$. When $\Delta_{1}=0$, system (10) has only one equilibrium point at $A_{0}\left(\phi_{0}, 0\right)$ in $\phi$-axis, where $\phi_{0}=-(1 / 2) c$. When $\Delta_{1}<0$, system (10) has not equilibrium point in $\phi$-axis. When $\Delta_{2}>0$, there exist two equilibrium points of system (10) in line $\phi=0$ at $S_{ \pm}\left(0, Y_{ \pm}\right), Y_{ \pm}= \pm \sqrt{\Delta_{2}}$. When $\Delta_{2}<0$, there is no equilibrium point of system (10) in line $\phi=0$.

Let $M\left(\phi_{e}, y_{e}\right)$ be the coefficient matrix of the linearized system of the system (10) at an equilibrium point $\left(\phi_{e}, y_{e}\right)$. Then we have

$$
M\left(\phi_{e}, y_{e}\right)=\left(\begin{array}{cc}
2 y_{e} & 2 \phi_{e} \\
-2 \phi_{e}-c & -2(k-1) y_{e}
\end{array}\right),
$$

and at this equilibrium point, we have

$$
\begin{gathered}
\operatorname{Trace}\left(M\left(\phi_{e}, y_{e}\right)\right)=-2(k-2) y_{e}, \\
J\left(\phi_{e}, y_{e}\right)=\operatorname{det} M\left(\phi_{e}, y_{e}\right)=-4(k-1) y_{e}^{2}+2 \phi_{e}\left(2 \phi_{e}^{2}+c\right) .
\end{gathered}
$$

By the theory of planar dynamical systems, we know that, for an equilibrium point $\left(\phi_{e}, y_{e}\right)$ of a planar integrable system, if $J\left(\phi_{e}, y_{e}\right)<0$, then the equilibrium point is a saddle point. If $J\left(\phi_{e}, y_{e}\right)>0$ and $\operatorname{Trace}\left(M\left(\phi_{e}, y_{e}\right)\right)=0$, then it is a center point. If $J\left(\phi_{e}, y_{e}\right)=0$ and the Poincaré index of the equilibrium point is zero, then it is a cusp.

For a fixed $h$, the level curve $H(\phi, y)=h$ defined by (11) determines a set of invariant curves of system (10) which contains different branches of curves. As $h$ is varied, it defines different families of orbits of (10) with different dynamical behaviors.

Using the property of equilibrium points and bifurcation theory, we obtain the following results.

When $k>1$, there are two bifurcation curves as follows:

$$
L_{1}: g=-\frac{1}{4} c^{2}, \quad L_{2}: g=0
$$

which divide the $(c, g)$-parameter plane into 10 subregions: $A_{1}=\left\{(c, g) \mid c<0, g<-(1 / 4) c^{2}\right\}, A_{2}=\{(c, g) \mid c<$ $\left.0, g=-(1 / 4) c^{2}\right\}, A_{3}=\left\{(c, g) c<0,-(1 / 4) c^{2}<g<0\right\}$, 
$A_{4}=\{(c, g) c<0, g=0\}, A_{5}=\{(c, g) c<0, g>0\}, A_{6}=$ $\{(c, g) c>0, g>0\}, A_{7}=\{(c, g) c>0, g=0\}, A_{8}=\{(c, g) c>$ $\left.0,-(1 / 4) c^{2}<g<0\right\}, A_{9}=\left\{(c, g) c>0, g=-(1 / 4) c^{2}\right\}$, and $A_{10}=\left\{(c, g) \mid c>0, g<-(1 / 4) c^{2}\right\}$.

When $k<-1$, there are three bifurcation curves as follows:

$$
L_{1}: g=-\frac{1}{4} c^{2}, \quad L_{2}: g=-\frac{k^{2}-1}{4 k^{2}} c^{2}, \quad L_{3}: g=0,
$$

which divide the $(c, g)$-parameter plane into 14 subregions: $B_{1}=\left\{(c, g) \mid c<0, g<-(1 / 4) c^{2}\right\}, B_{2}=\{(c, g) \mid c<$ $\left.0, g=-(1 / 4) c^{2}\right\}, B_{3}=\left\{(c, g) \mid c<0,-(1 / 4) c^{2}<g<-\left(\left(k^{2}-\right.\right.\right.$ 1) $\left.\left./ 4 k^{2}\right) c^{2}\right\}, B_{4}=\left\{(c, g) \mid c<0, g=-\left(\left(k^{2}-1\right) / 4 k^{2}\right) c^{2}\right\}$, $B_{5}=\left\{(c, g) \mid c<0,-\left(\left(k^{2}-1\right) / 4 k^{2}\right) c^{2}<g<0\right\}, B_{6}=\{(c, g) \mid$ $c<0, g=0\}, B_{7}=\{(c, g) \mid c<0, g>0\}, B_{8}=\{(c, g) \mid$ $c>0, g>0\}, B_{9}=\{(c, g) \mid c>0, g=0\}, B_{10}=\{(c, g) \mid$ $\left.c>0,-\left(\left(k^{2}-1\right) / 4 k^{2}\right) c^{2}<g<0\right\}, B_{11}=\{(c, g) \mid c>0, g=$ $\left.-\left(\left(k^{2}-1\right) / 4 k^{2}\right) c^{2}\right\}, B_{12}=\left\{(c, g) \mid c>0,-(1 / 4) c^{2}<g<\right.$ $\left.-\left(\left(k^{2}-1\right) / 4 k^{2}\right) c^{2}\right\}, B_{13}=\left\{(c, g) \mid c>0, g=-(1 / 4) c^{2}\right\}$, and $B_{14}=\left\{(c, g) \mid c>0, g<-(1 / 4) c^{2}\right\}$.

The bifurcation sets and phase portraits of system (10) are shown in Figures 1 and 2.

\section{Exact Peakon, Solitary, and Smooth Periodic Wave Solutions of (6)}

In this section, we present some exact peakon, solitary, and smooth periodic wave solutions of (6) through some special phase orbits. Next, we always suppose that

$$
\begin{gathered}
h_{1}=\frac{1}{24}\left(c-\sqrt{\Delta_{1}}\right)\left(c^{2}-c \sqrt{\Delta_{1}}+8 g\right), \\
h_{2}=\frac{1}{24}\left(c+\sqrt{\Delta_{1}}\right)\left(c^{2}+c \sqrt{\Delta_{1}}+8 g\right), \\
h_{3}=\frac{2\left(3 c^{2}-3 c \sqrt{\Delta_{1}}+8 g\right)}{3\left(c-\sqrt{\Delta_{1}}\right)^{3}}, \quad h_{4}=\frac{2\left(3 c^{2}+3 c \sqrt{\Delta_{1}}+8 g\right)}{3\left(c+\sqrt{\Delta_{1}}\right)^{3}} .
\end{gathered}
$$

\subsection{Peakon Solutions}

(i) For given $k=-2$ and $h=2 / 3 c$ in Figure 2(b), the level curve is shown in Figure 3(e). From Figure 3(e), we see that there are two heteroclinic orbits connecting with complex equilibrium points $(0, \pm(\sqrt{3} / 6) c)$ and a cusp $\left(\phi_{0}, 0\right)$ of systems (9) and (10) when $(c, g) \in B_{2}$, where $\phi_{0}=-(1 / 2) c$. Their expressions are

$$
y= \pm \sqrt{-\frac{2}{3 c}}\left(\phi_{0}-\phi\right) \sqrt{\phi_{0}-\phi}, \quad 0 \leq \phi<\phi_{0} .
$$

Substituting (20) into $d \phi / d \xi=y$ and integrating it along the heteroclinic orbits yield the equation

$$
\int_{0}^{\phi} \frac{d s}{\left(\phi_{0}-s\right) \sqrt{\phi_{0}-s}}=\sqrt{-\frac{2}{3 c}}|\xi| .
$$

Completing the above integral and solving the equation for $\phi$, it follows that

$$
\phi(\xi)=\phi_{0}-\frac{1}{\left(\omega|\xi|+1 / \sqrt{\phi_{0}}\right)^{2}},
$$

where $\omega=(1 / 2) \sqrt{-2 / 3 c}$.

Noting that $u(x, t)=\phi(\xi)$ and $\xi=x-c t$, we get a peakon solution as follows:

$$
u(x, t)=\phi_{0}-\frac{1}{\left(\omega|x-c t|+1 / \sqrt{\phi_{0}}\right)^{2}} .
$$

The profile of (23) is shown in Figure 4(a).

(ii) For given $k<-1$ and $h=0$ in Figure 2(d), the level curve is shown in Figure 3(h). From Figure 3(h), we see that there are two heteroclinic orbits connecting with complex equilibrium points $(0, \pm(c / 2 k) \sqrt{-(k+1)})$ and a saddle point $\left(\phi_{2}, 0\right)$ of systems $(9)$ and $(10)$ when $(c, g) \in B_{4}$, where $\phi_{2}=$ $-((k+1) / 2 k) c$. Their expressions are

$$
y= \pm \sqrt{-\frac{1}{k+1}}\left(\phi_{2}-\phi\right), \quad 0 \leq \phi<\phi_{2} .
$$

Substituting (24) into the $d \phi / d \xi=y$ and integrating it along the heteroclinic orbits yield the equation

$$
\int_{0}^{\phi} \frac{d s}{\phi_{2}-s}=\sqrt{-\frac{1}{k+1}}|\xi| .
$$

Completing the above integral, we can get a peakon solution as follows:

$$
u(x, t)=\phi_{2}\left(1-e^{-\omega|x-c t|}\right),
$$

where $\omega=\sqrt{-1 /(k+1)}$. The profile of (26) is shown in Figure 4(b).

(iii) For given $k<-1$ and $h=0$ in Figure 2(k), the level curve is shown in Figure 3(m). From Figure 3(m), we see that there are two heteroclinic orbits connecting with complex equilibrium points $(0, \pm(c / 2 k) \sqrt{-(k+1)})$ and a saddle point $\left(\phi_{1}, 0\right)$ of systems $(9)$ and $(10)$ when $(c, g) \in B_{11}$, where $\phi_{1}=-((k+1) / 2 k) c$. Their expressions are

$$
y= \pm \sqrt{-\frac{1}{k+1}}\left(\phi-\phi_{1}\right), \quad \phi_{1}<\phi \leq 0 .
$$

Substituting (27) into $d \phi / d \xi=y$ and integrating it along the heteroclinic orbits yield the equation

$$
\int_{\phi}^{0} \frac{d s}{s-\phi_{1}}=\sqrt{-\frac{1}{k+1}}|\xi|
$$

Completing the above integral, we can get a peakon solution as follows:

$$
u(x, t)=\phi_{1}+\left(1-e^{-\omega|x-c t|}\right),
$$

where $\omega=\sqrt{-1 /(k+1)}$. The profile of (29) is shown in Figure 4(c). 


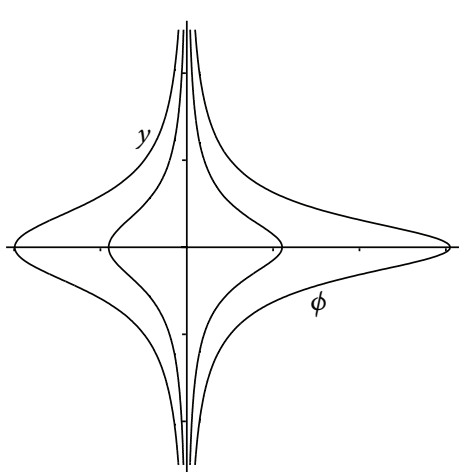

(a) $(c, g) \in A_{1}$

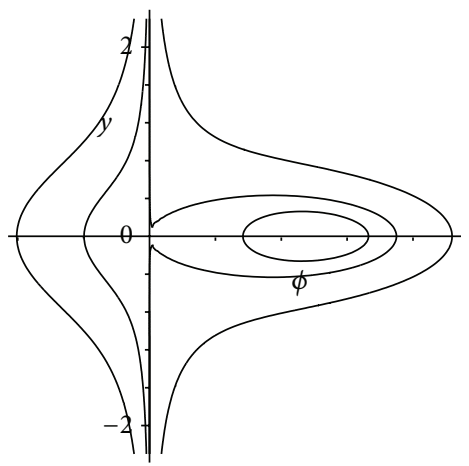

(d) $(c, g) \in A_{4}$

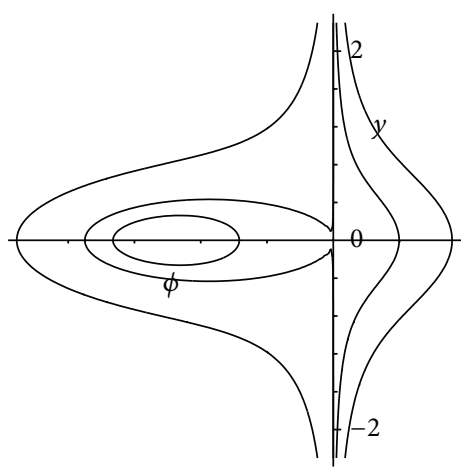

(g) $(c, g) \in A_{7}$

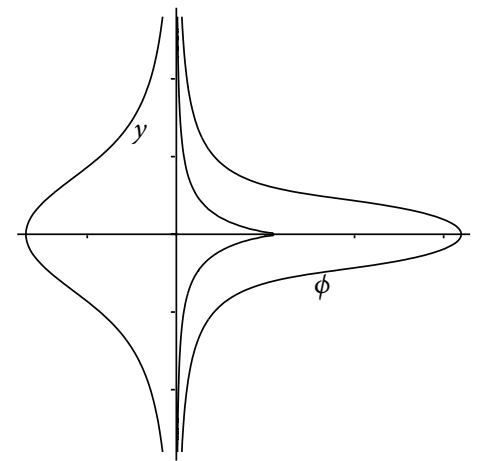

(b) $(c, g) \in A_{2}$

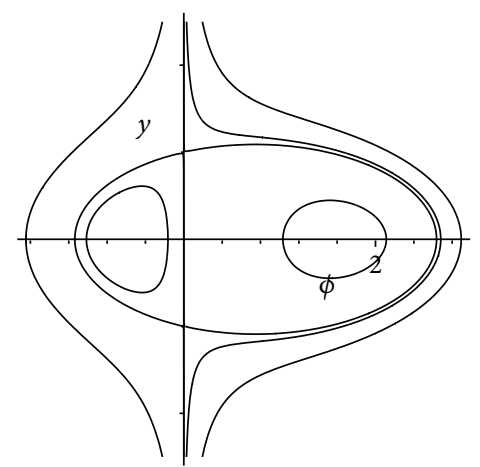

(e) $(c, g) \in A_{5}$

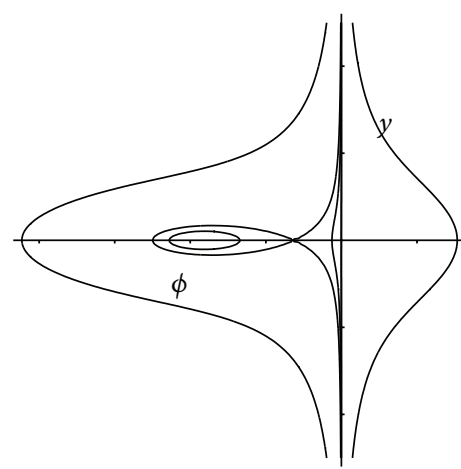

(h) $(c, g) \in A_{8}$

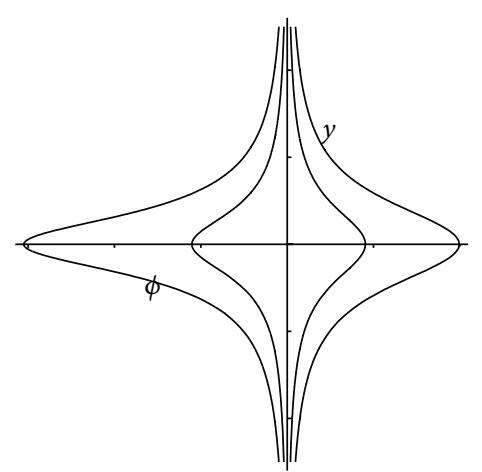

(j) $(c, g) \in A_{10}$

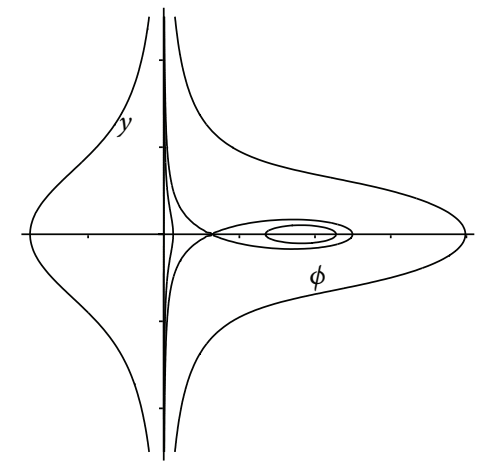

(c) $(c, g) \in A_{3}$

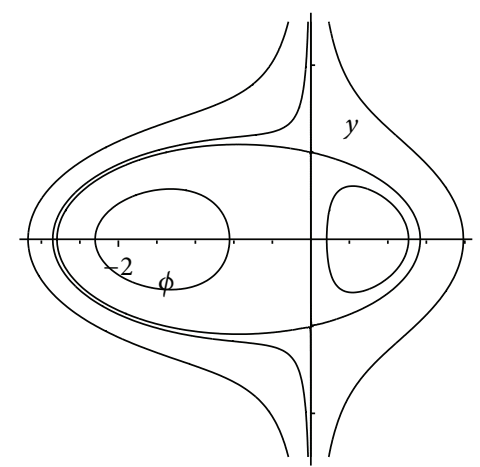

(f) $(c, g) \in A_{6}$

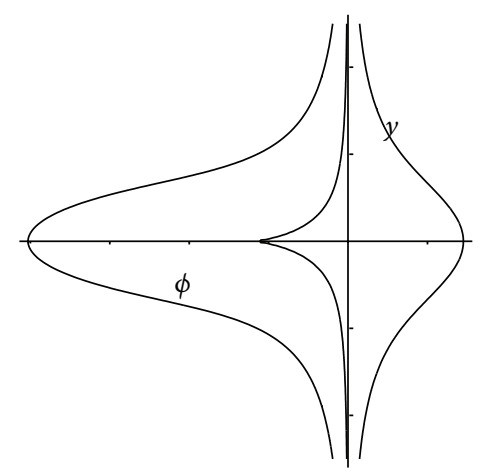

(i) $(c, g) \in A_{9}$

FIGURE 1: The bifurcation sets and phase portraits of system (10) for $k>1$. 


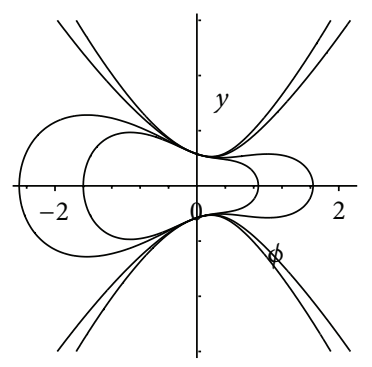

(a) $(c, g) \in B_{1}$

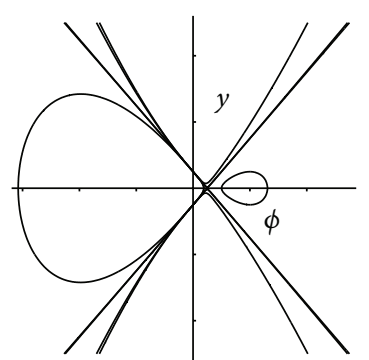

(d) $(c, g) \in B_{4}$

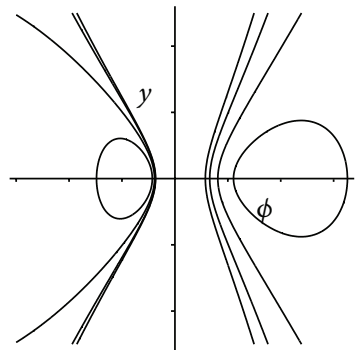

(g) $(c, g) \in B_{7}$

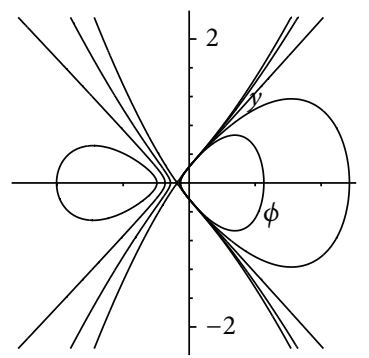

(j) $(c, g) \in B_{10}$

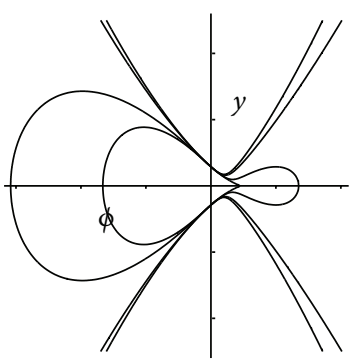

(b) $(c, g) \in B_{2}$

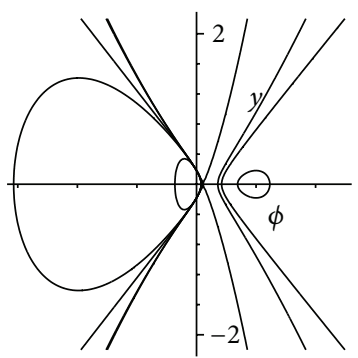

(e) $(c, g) \in B_{5}$

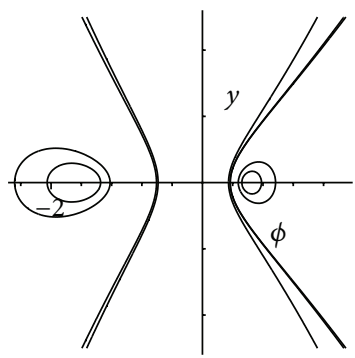

(h) $(c, g) \in B_{8}$

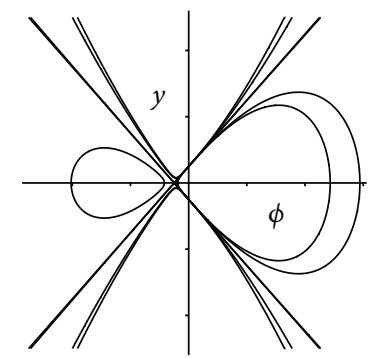

(k) $(c, g) \in B_{11}$

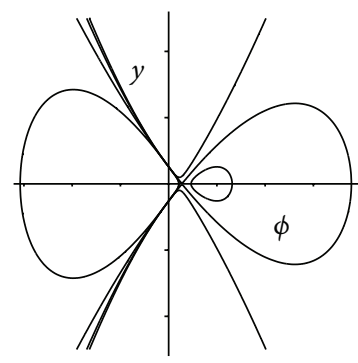

(c) $(c, g) \in B_{3}$

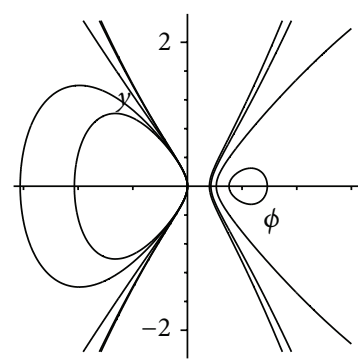

(f) $(c, g) \in B_{6}$

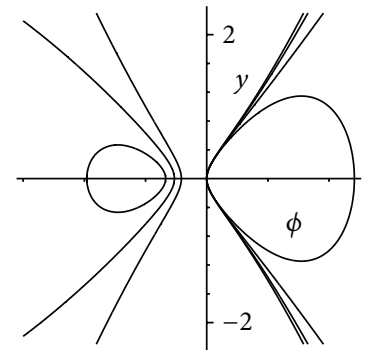

(i) $(c, g) \in B_{9}$

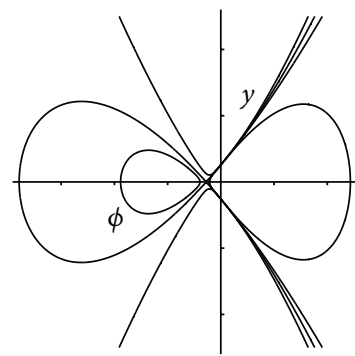

(l) $(c, g) \in B_{12}$

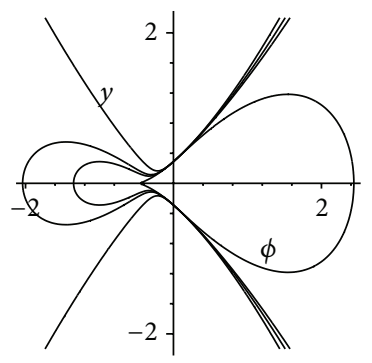

(m) $(c, g) \in B_{13}$

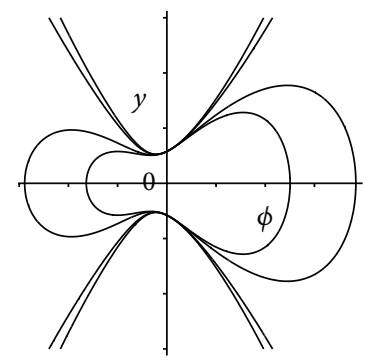

(n) $(c, g) \in B_{14}$

FIGURE 2: The bifurcation sets and phase portrait of system (10) as for $k<-1$. 


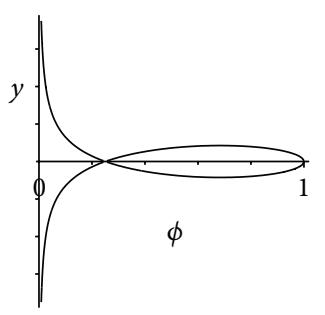

(a) $h_{*}=h_{2}$

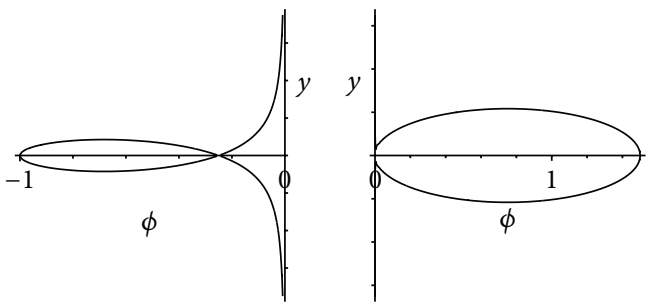

(c) $h_{*}=0$

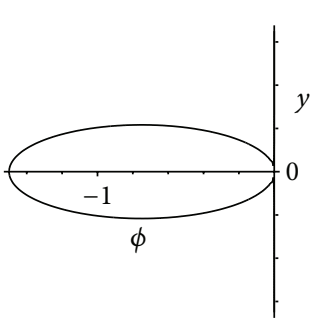

(d) $h_{*}=0$

(b) $h_{*}=h_{1}$

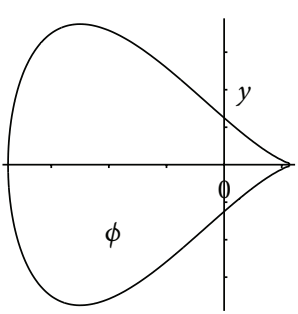

(f) $h_{*}=-1 / 3 c^{2}$

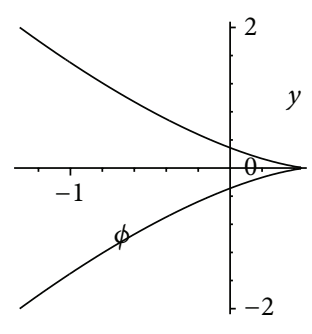

(e) $h_{*}=2 / 3 c$

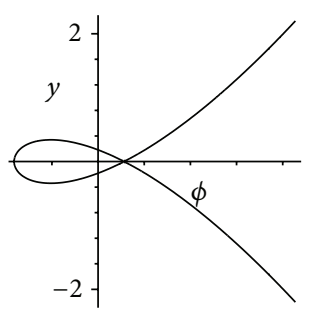

(i) $h_{*}=h_{4}$

(g) $h_{*}=h_{4}$

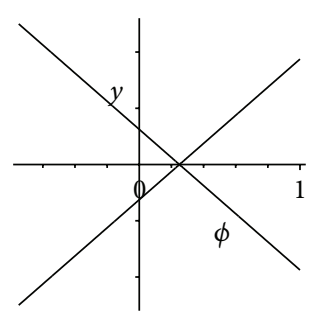

(h) $h_{*}=0$

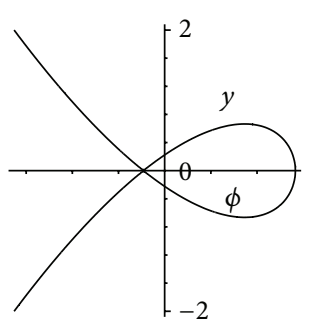

(l) $h_{*}=h_{3}$

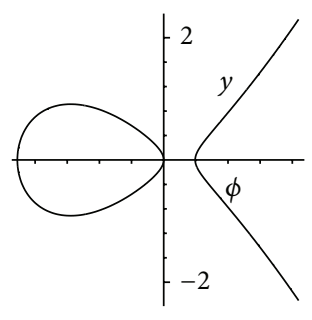

(j) $h_{*}>0$

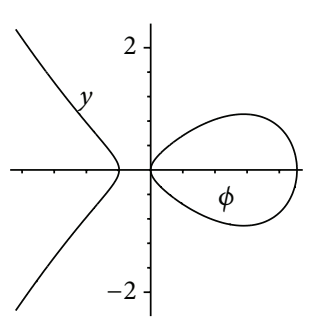

(k) $h_{*}<0$

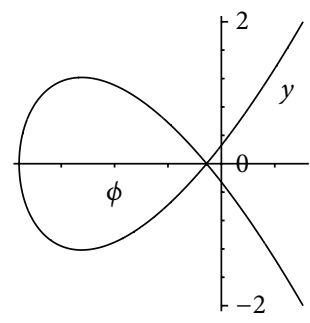

(n) $h_{*}=h_{3}$

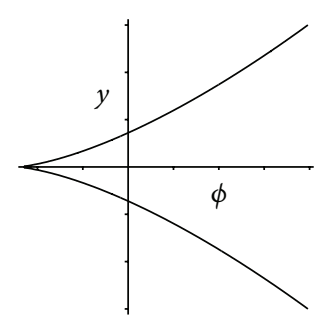

(o) $h_{*}=2 / 3 c$

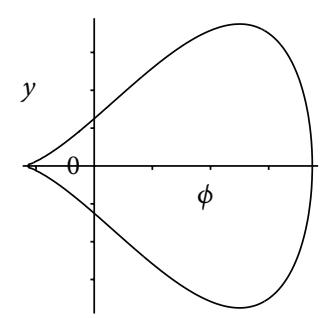

(p) $h_{*}=-1 / 3 c^{2}$

FIgURE 3: The level curves of system (10) defined by $h=h_{*}$.

(iv) For given $k=-2$ and $h=2 / 3 c$ in Figure 2(m), the level curve is shown in Figure 3(o). From Figure 3(o), we see that there are two heteroclinic orbits connecting with complex equilibrium points $(0, \pm(\sqrt{3} / 6) c)$ and a cusp $\left(\phi_{0}, 0\right)$ of systems (9) and (10) when $(c, g) \in B_{13}$, where $\phi_{0}=-(1 / 2) c$. Their expressions are

$$
y= \pm \sqrt{\frac{2}{3 c}}\left(\phi-\phi_{0}\right) \sqrt{\phi-\phi_{0}}, \quad \phi_{0}<\phi \leq 0 .
$$




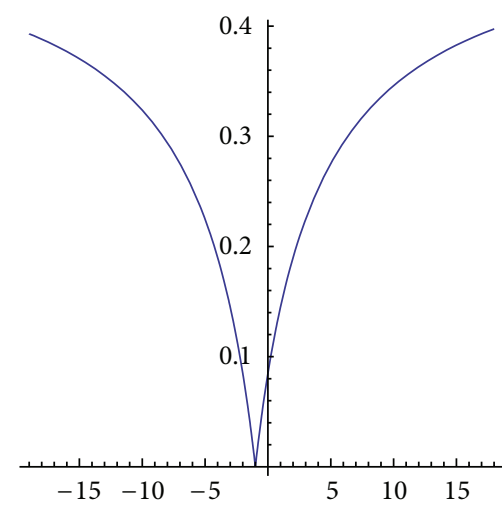

(a) $c=-1, g=-1 / 4$

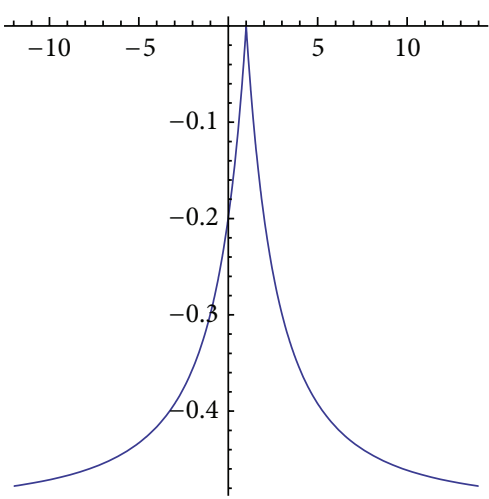

(d) $c=1, g=-1 / 4$

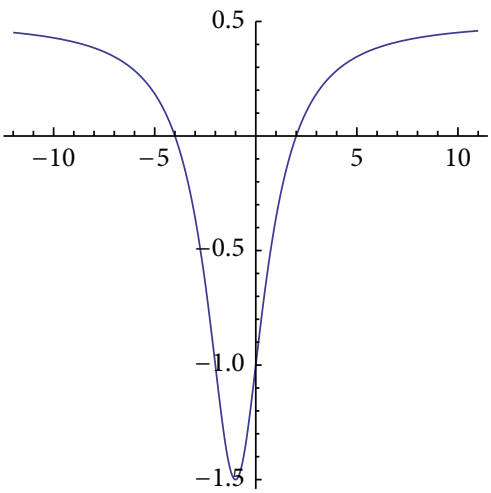

(g) $c=-1, g=-1 / 4$

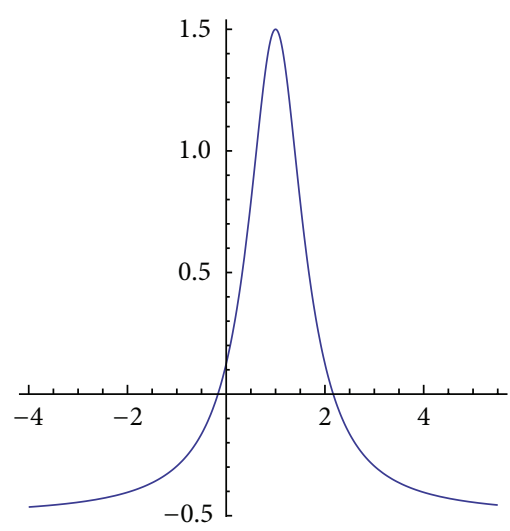

(j) $c=1, g=-1 / 4$

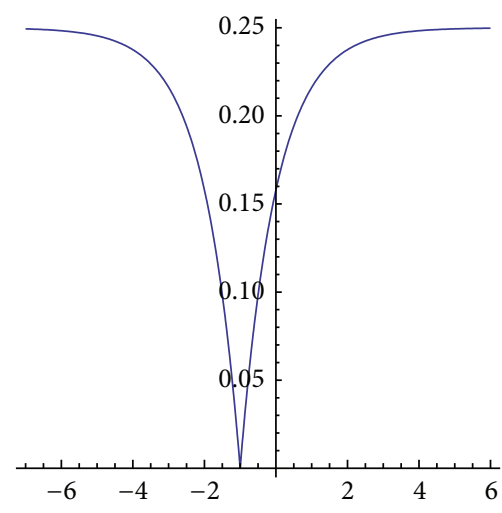

(b) $k=-2, c=-1, g=-3 / 16$

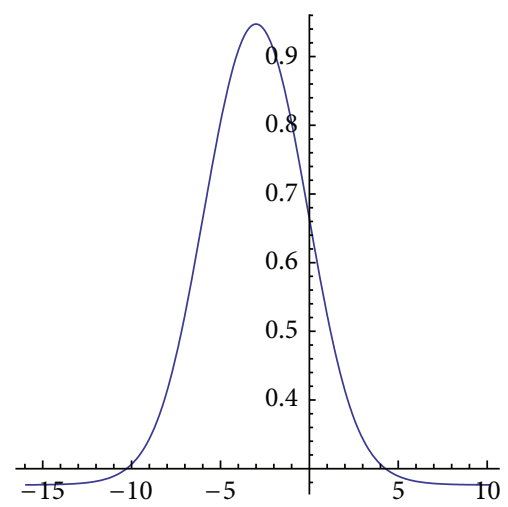

(e) $c=-1, g=-1 / 5$

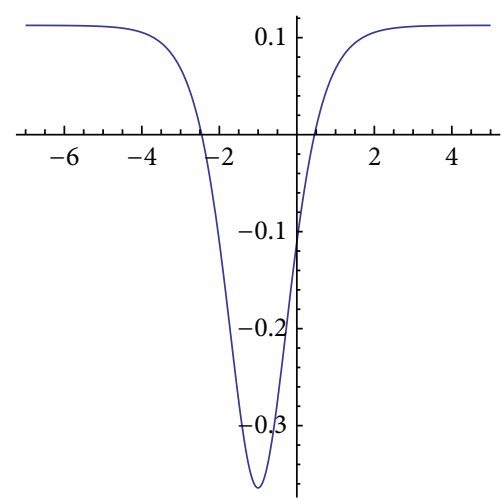

(h) $c=-1, g=-0.1$

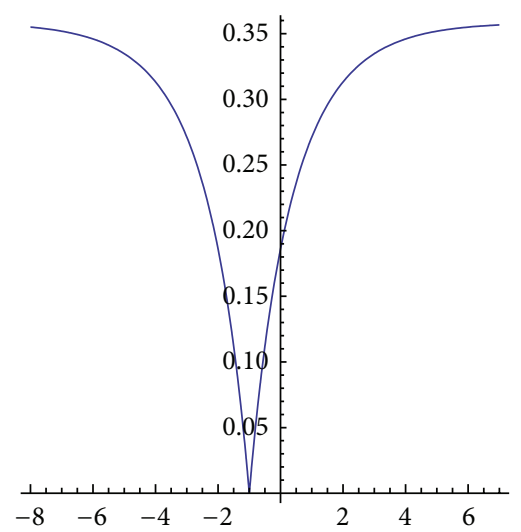

(k) $c=-1, g=-0.23$

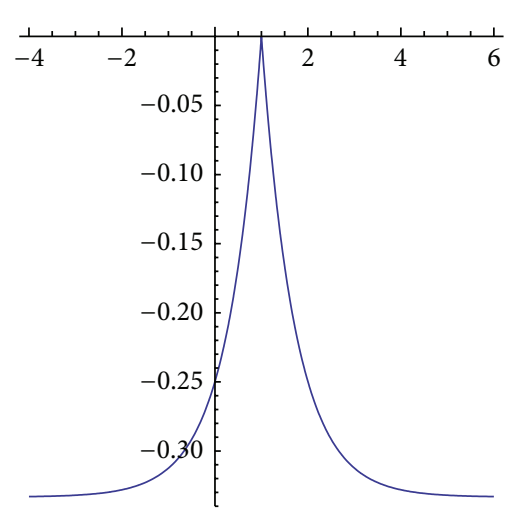

(c) $k=-3, c=1, g=-2 / 9$

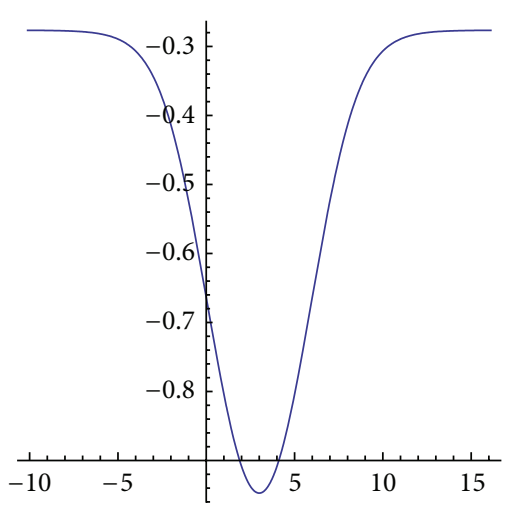

(f) $c=-1, g=-1 / 5$

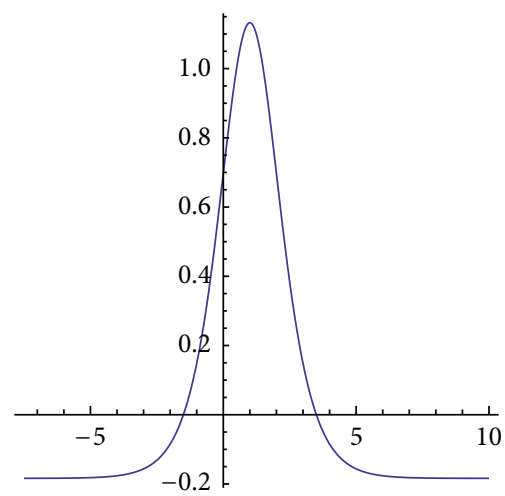

(i) $c=1, g=-0.15$

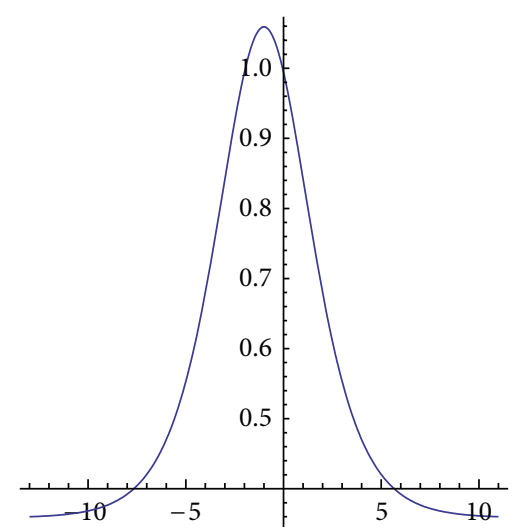

(l) $c=-1, g=-0.23$

Figure 4: Continued. 


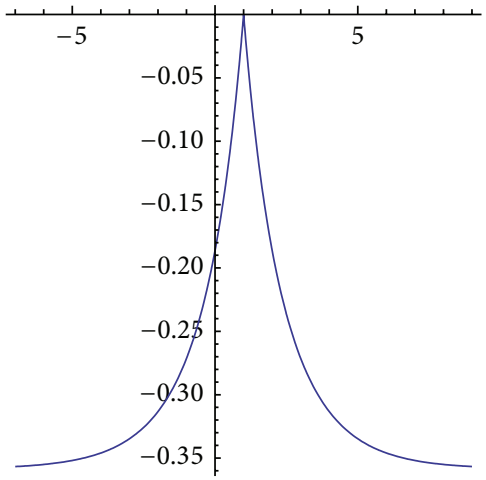

(m) $c=1, g=-0.23$

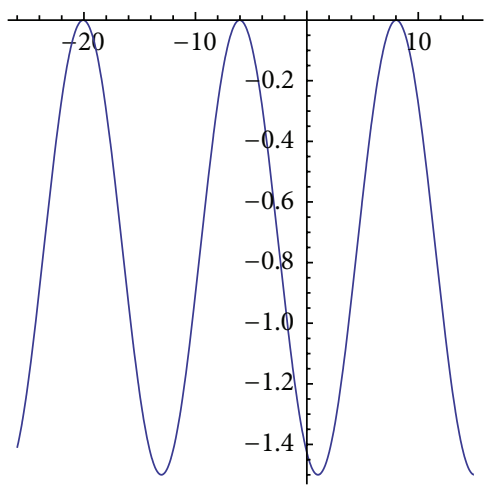

(p) $c=1, k=4$

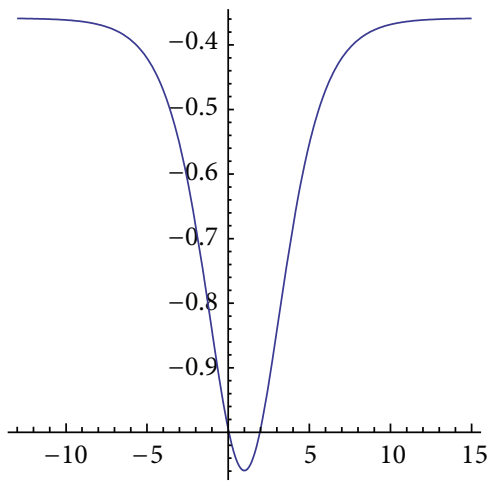

(n) $c=1, g=-0.23$

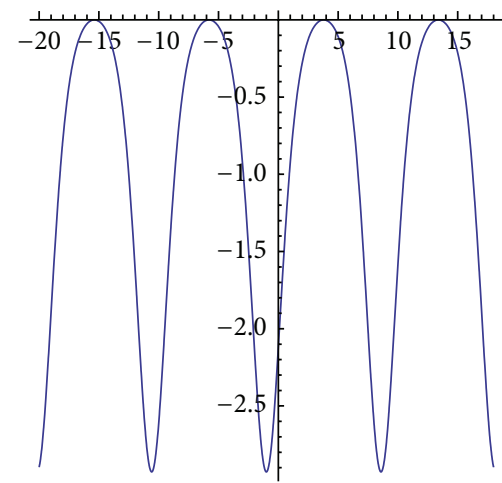

(q) $c=-1, h=0.4$

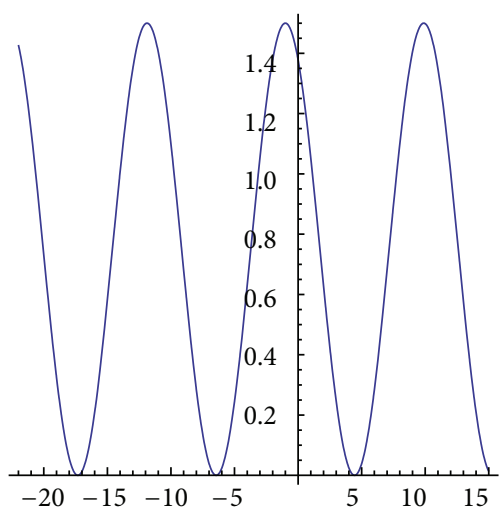

(o) $c=-1, k=2$

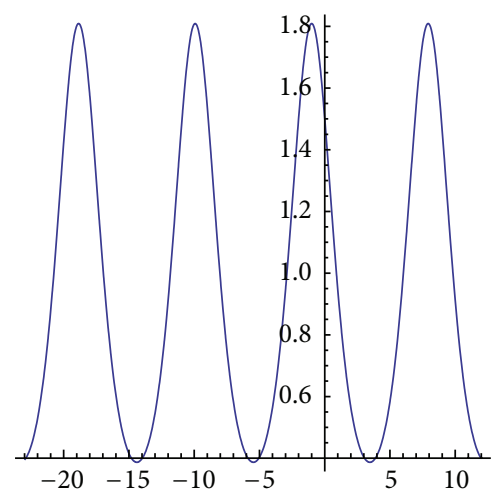

(r) $c=1, h=-0.7$

FIGURE 4: Peakons, solitary, and smooth periodic waves of (6) for $x=1$.

Substituting (30) into $d \phi / d \xi=y$ and integrating it along the heteroclinic orbits yield the equation

$$
\int_{\phi}^{0} \frac{d s}{\left(s-\phi_{0}\right) \sqrt{s-\phi_{0}}}=\sqrt{\frac{2}{3 c}}|\xi| .
$$

Completing the above integral, we can get a peakon solution as follows:

$$
u(x, t)=\phi_{0}+\frac{1}{\left(\omega|x-c t|+1 / \sqrt{-\phi_{0}}\right)^{2}},
$$

where $\omega=(1 / 2) \sqrt{2 / 3 c}$. The profile of (32) is shown in Figure 4(d).

\subsection{Solitary Wave Solutions}

(i) For given $k=2$ and $h=h_{2}$ in Figure 1(c), the level curve is shown in Figure 3(a). From Figure 3(a), we see that there is a homoclinic orbit connecting with a saddle point $\left(\phi_{2}, 0\right)$ of systems (9) and (10) and passing point $\left(\phi_{M}, 0\right)$ when $(c, g) \in$ $A_{3}$, where $\phi_{2}=-\left(c-\sqrt{\Delta_{1}}\right) / 2$ and $\phi_{M}=-(1 / 2) c+\sqrt{\Delta_{1}}$. Its expression is

$$
y= \pm \frac{\phi-\phi_{2}}{\sqrt{3} \phi} \sqrt{\phi\left(\phi_{M}-\phi\right)}, \quad \phi_{2}<\phi \leq \phi_{M} .
$$

Substituting (33) into $d \phi / d \xi=y$ and integrating it along the homoclinic orbit yield the equation

$$
\int_{\phi}^{\phi_{M}} \frac{s d s}{\left(s-\phi_{2}\right) \sqrt{s\left(\phi_{M}-s\right)}}=\frac{\sqrt{3}}{3}|\xi| .
$$

Completing the above integral, we can get a solitary wave solution as follows:

$$
\begin{gathered}
u(x, t)=\frac{\phi_{2} \phi_{M} \cosh ^{2}(\omega \tau)}{\phi_{2}+\phi_{M} \sinh ^{2}(\omega \tau)}, \\
x(\tau)=\sqrt{3}\left(\tau+2 \tan ^{-1}\left(\sqrt{\frac{\phi_{M}-\phi_{2}}{\phi_{2}}} \tanh (\omega \tau)\right)\right)+c t,
\end{gathered}
$$

where $\omega=(1 / 2) \sqrt{\left(\phi_{M}-\phi_{2}\right) / \phi_{2}}$ and $\tau$ is a new parametric variable. The profile of (35) is shown in Figure 4(e).

(ii) For given $k=2$ and $h=h_{1}$ in Figure 1(h), the level curve is shown in Figure 3(b). From Figure 3(b), we see that there is a homoclinic orbit connecting with a saddle point $\left(\phi_{1}, 0\right)$ of systems $(9)$ and $(10)$ and passing point $\left(\phi_{m}, 0\right)$ when $(c, g) \in A_{8}$, where $\phi_{1}=\left(-c+\sqrt{\Delta_{1}}\right) / 2$ and $\phi_{m}=-(1 / 2) c-$ $\sqrt{\Delta_{1}}$. Its expression is

$$
y= \pm \frac{\phi_{1}-\phi}{\sqrt{3} \phi} \sqrt{\phi\left(\phi_{m}-\phi\right)}, \quad \phi_{m} \leq \phi<\phi_{1} .
$$


Substituting (36) into $d \phi / d \xi=y$ and integrating it along the homoclinic orbit yield the equation

$$
\int_{\phi_{m}}^{\phi} \frac{s d s}{\left(\phi_{1}-s\right) \sqrt{s\left(\phi_{m}-s\right)}}=-\frac{\sqrt{3}}{3}|\xi| .
$$

Completing the above integral, we can get a solitary wave solution as follows:

$$
\begin{gathered}
u(x, t)=\frac{\phi_{1} \phi_{m} \cosh ^{2}(\omega \tau)}{\phi_{1}+\phi_{m} \sinh ^{2}(\omega \tau)}, \\
x(\tau)=\sqrt{3}\left(\tau+2 \tan ^{-1}\left(\sqrt{\frac{\phi_{m}-\phi_{1}}{\phi_{1}}} \tanh (\omega \tau)\right)\right)+c t,
\end{gathered}
$$

where $\omega=(1 / 2) \sqrt{\left(\phi_{m}-\phi_{1}\right) / \phi_{1}}$ and $\tau$ is a new parametric variable. The profile of (38) is shown in Figure 4(f).

(iii) For given $k=-3$ and $h=-1 / 3 c^{2}$ in Figure 2(b), the level curve is shown in Figure 3(f). From Figure 3(f), we see that there is a homoclinic orbit connecting with complex equilibrium points $(0, \pm(1 / 4) c)$ and a cusp $\left(\phi_{0}, 0\right)$ of systems (9) and (10) and passing point $\left(\phi_{m}, 0\right)$ when $(c, g) \in B_{2}$, where $\phi_{0}=-(1 / 2) c$ and $\phi_{m}=(3 / 2) c$. Its expression is

$$
y= \pm \frac{1}{\sqrt{3} c}\left(\phi_{0}-\phi\right) \sqrt{\left(\phi-\phi_{m}\right)\left(\phi_{0}-\phi\right)}, \quad \phi_{m} \leq \phi<\phi_{0} .
$$

Substituting (39) into $d \phi / d \xi=y$ and integrating it along the homoclinic orbit yield the equation

$$
\int_{\phi_{m}}^{\phi} \frac{d s}{\left(\phi_{0}-s\right) \sqrt{\left(s-\phi_{m}\right)\left(\phi_{0}-s\right)}}=-\frac{1}{\sqrt{3} c}|\xi| .
$$

Completing the above integral, we can get a solitary wave solution as follows:

$$
u(x, t)=\frac{\phi_{m}+\phi_{0}(\omega(x-c t))^{2}}{1+(\omega(x-c t))^{2}},
$$

where $\omega=\left(\phi_{m}-\phi_{0}\right) / 2 c \sqrt{3}$. The profile of (41) is shown in Figure $4(\mathrm{~g})$.

(iv) For given $k=-2$ and $h=h_{4}$ in Figure 2(e), the level curve is shown in Figure 3(i). From Figure 3(i), we see that there is a homoclinic orbit connecting with complex equilibrium points $(0, \pm \sqrt{-3 g} / 3)$ and a saddle point $\left(\phi_{2}, 0\right)$ of systems (9) and (10) and passing point $\left(\phi_{m}, 0\right)$ when $(c, g) \in$ $B_{5}$, where $\phi_{2}=-\left(c-\sqrt{\Delta_{1}}\right) / 2$ and $\phi_{m}=2 g\left(c+\sqrt{\Delta_{1}}\right) /\left(3 c^{2}+\right.$ $3 c \sqrt{\Delta_{1}}+8 g$ ). Its expression is

$$
y= \pm \sqrt{\Omega}\left(\phi_{2}-\phi\right) \sqrt{\phi-\phi_{m}}, \quad \phi_{m} \leq \phi<\phi_{2},
$$

where $\Omega=2\left(3 c^{2}+3 c \sqrt{\Delta_{1}}+8 g\right) / 3\left(c+\sqrt{\Delta_{1}}\right)^{3}$.

Substituting (42) into $d \phi / d \xi=y$ and integrating it along the homoclinic orbit yield the equation

$$
\int_{\phi_{m}}^{\phi} \frac{d s}{\left(\phi_{2}-s\right) \sqrt{s-\phi_{m}}}=\sqrt{\Omega}|\xi| .
$$

Completing the above integral, we can get a solitary wave solution as follows:

$$
u(x, t)=\phi_{m}+\left(\phi_{2}-\phi_{m}\right) \tanh ^{2}(\omega(x-c t)),
$$

where $\omega=(1 / 2) \sqrt{\Omega\left(\phi_{2}-\phi_{m}\right)}$. The profile of $(44)$ is shown in Figure 4(h).

(v) For given $k=-2$ and $h=h_{3}$ in Figure $2(\mathrm{j})$, the level curve is shown in Figure 3(1). From Figure 3(l), we see that there is a homoclinic orbit connecting with complex equilibrium points $(0, \pm \sqrt{-3 g} / 3)$ and a saddle point $\left(\phi_{1}, 0\right)$ of systems (9) and (10) and passing point $\left(\phi_{M}, 0\right)$ when $(c, g) \epsilon$ $B_{10}$, where $\phi_{1}=-\left(c+\sqrt{\Delta_{1}}\right) / 2$ and $\phi_{M}=2 g\left(c-\sqrt{\Delta_{1}}\right) /\left(3 c^{2}-\right.$ $3 c \sqrt{\Delta_{1}}+8 g$ ). Its expression is

$$
y= \pm \sqrt{\Omega}\left(\phi-\phi_{1}\right) \sqrt{\phi_{M}-\phi}, \quad \phi_{1}<\phi \leq \phi_{M}
$$

where $\Omega=2\left(3 c^{2}-3 c \sqrt{\Delta_{1}}+8 g\right) / 3\left(-c+\sqrt{\Delta_{1}}\right)^{3}$.

Substituting (45) into $d \phi / d \xi=y$ and integrating it along the homoclinic orbit yield the equation

$$
\int_{\phi}^{\phi_{M}} \frac{d s}{\left(s-\phi_{1}\right) \sqrt{\phi_{M}-s}}=\sqrt{\Omega}|\xi| .
$$

Completing the above integral, we can get a solitary wave solution as follows:

$$
u(x, t)=\phi_{M}+\left(\phi_{1}-\phi_{M}\right) \tanh ^{2}(\omega(x-c t))
$$

where $\omega=(1 / 2) \sqrt{\Omega\left(\phi_{M}-\phi_{1}\right)}$. The profile of (47) is shown in Figure 4(i).

(vi) For given $k=-3$ and $h=-1 / 3 c^{2}$ in Figure 2(m), the level curve is shown in Figure 3(p). From Figure 3(p), we see that there is a homoclinic orbit connecting with complex equilibrium points $(0, \pm(1 / 4) c)$ and a cusp $\left(\phi_{0}, 0\right)$ of systems (9) and (10) and passing point $\left(\phi_{M}, 0\right)$ when $(c, g) \in B_{13}$, where $\phi_{0}=-(1 / 2) c$ and $\phi_{M}=(3 / 2) c$. Its expression is

$$
y= \pm \frac{1}{\sqrt{3} c}\left(\phi-\phi_{0}\right) \sqrt{\left(\phi-\phi_{0}\right)\left(\phi_{M}-\phi\right)}, \quad \phi_{0}<\phi \leq \phi_{M} .
$$

Substituting (48) into $d \phi / d \xi=y$ and integrating it along the homoclinic orbit yield the equation

$$
\int_{\phi}^{\phi_{M}} \frac{d s}{\left(s-\phi_{0}\right) \sqrt{\left(s-\phi_{0}\right)\left(\phi_{M}-s\right)}}=\frac{1}{\sqrt{3} c}|\xi| .
$$

Completing the above integral, we can get a solitary wave solution as follows:

$$
u(x, t)=\frac{\phi_{M}+\phi_{0}(\omega(x-c t))^{2}}{1+(\omega(x-c t))^{2}},
$$

where $\omega=\left(\phi_{M}-\phi_{0}\right) / 2 c \sqrt{3}$. The profile of (50) is shown in Figure 4(j). 
3.3. Coexistence of Peakon and Solitary Wave Solutions. From Figures 2(c) and 2(l), we easily see the coexistence of peakon and solitary wave for (6) when $k<-1$ and $(c, g) \in B_{3}$ (or when $k<-1$ and $\left.(c, g) \in B_{12}\right)$. Here, we give only several exact parametric representations of these waves.

(i) For given $k=-2$ and $h=h_{4}$ in Figure 2(c), the level curve is shown in Figure 3(g). From Figure 3(g), we see that there are two heteroclinic orbits connecting with complex equilibrium points $(0, \pm \sqrt{-3 g} / 3)$ and a saddle point $\left(\phi_{2}, 0\right)$ of systems (9) and (10), and at the same time, there is a homoclinic orbit connecting with the saddle point $\left(\phi_{2}, 0\right)$ and passing point $\left(\phi_{M}, 0\right)$ when $(c, g) \in B_{3}$, where $\phi_{2}=-(c-$ $\left.\sqrt{\Delta_{1}}\right) / 2$ and $\phi_{M}=2 g\left(c+\sqrt{\Delta_{1}}\right) /\left(3 c^{2}+3 c \sqrt{\Delta_{1}}+8 g\right)$. Their expressions are, respectively,

$$
\begin{gathered}
y= \pm \sqrt{\Omega}\left(\phi_{2}-\phi\right) \sqrt{\phi_{M}-\phi}, \quad 0 \leq \phi<\phi_{2}, \\
y= \pm \sqrt{\Omega}\left(\phi-\phi_{2}\right) \sqrt{\phi_{M}-\phi}, \quad \phi_{2}<\phi \leq \phi_{M},
\end{gathered}
$$

where $\Omega=-2\left(3 c^{2}+3 c \sqrt{\Delta_{1}}+8 g\right) / 3\left(c+\sqrt{\Delta_{1}}\right)^{3}$.

Substituting (51) into $(d \phi / d \xi)=y$ and integrating it along the heteroclinic orbits yield the equation

$$
\int_{0}^{\phi} \frac{d s}{\left(\phi_{2}-s\right) \sqrt{\phi_{M}-s}}=\sqrt{\Omega}|\xi| .
$$

Completing the above integral, we can get a peakon solution as follows:

$$
=\frac{\phi_{2}-\phi_{M} \operatorname{sech}^{2}\left(\omega|x-c t|+\tanh ^{-1} \sqrt{\left(\phi_{M}-\phi_{2}\right) / \phi_{M}}\right)}{\tanh ^{2}\left(\omega|x-c t|+\tanh ^{-1} \sqrt{\left(\phi_{M}-\phi_{2}\right) / \phi_{M}}\right)},
$$

where $\omega=(1 / 2) \sqrt{\Omega\left(\phi_{M}-\phi_{2}\right)}$. The profile of (54) is shown in Figure 4(k).

Substituting (52) into $d \phi / d \xi=y$ and integrating it along the homoclinic orbit yield the equation

$$
\int_{\phi}^{\phi_{M}} \frac{d s}{\left(s-\phi_{2}\right) \sqrt{\phi_{M}-s}}=\sqrt{\Omega}|\xi| .
$$

Completing the above integral, we can get a solitary wave solution as follows:

$$
u(x, t)=\phi_{M}-\left(\phi_{M}-\phi_{2}\right) \tanh ^{2}(\omega(x-c t)),
$$

where $\omega=(1 / 2) \sqrt{\Omega\left(\phi_{M}-\phi_{2}\right)}$. The profile of (56) is shown in Figure 4(l).

(ii) For given $k=-2$ and $h=h_{3}$ in Figure 2(l), the level curve is shown in Figure 3(n). From Figure 3(n), we see that there are two heteroclinic orbits connecting with complex equilibrium points $(0, \pm \sqrt{-3 g} / 3)$ and a saddle point $\left(\phi_{1}, 0\right)$ of systems (9) and (10) and, at the same time, there is a homoclinic orbit connecting with the saddle point $\left(\phi_{1}, 0\right)$ and passing point $\left(\phi_{m}, 0\right)$ when $(c, g) \in B_{12}$, where $\phi_{1}=-\left(c+\sqrt{\Delta_{1}}\right) / 2$ and $\phi_{m}=2 g\left(c-\sqrt{\Delta_{1}}\right) /\left(3 c^{2}-\right.$ $\left.3 c \sqrt{\Delta_{1}}+8 g\right)$. Their expressions are, respectively,

$$
\begin{gathered}
y= \pm \sqrt{\Omega}\left(\phi-\phi_{1}\right) \sqrt{\phi-\phi_{m}}, \quad \phi_{1}<\phi \leq 0, \\
y= \pm \sqrt{\Omega}\left(\phi_{1}-\phi\right) \sqrt{\phi-\phi_{m}}, \quad \phi_{m} \leq \phi<\phi_{1},
\end{gathered}
$$

where $\Omega=2\left(3 c^{2}-3 c \sqrt{\Delta_{1}}+8 g\right) / 3\left(c-\sqrt{\Delta_{1}}\right)^{3}$.

Substituting (57) into $d \phi / d \xi=y$ and integrating it along the heteroclinic orbits yield the equation

$$
\int_{\phi}^{0} \frac{d s}{\left(s-\phi_{1}\right) \sqrt{s-\phi_{m}}}=\sqrt{\Omega}|\xi| .
$$

Completing the above integral, we can get a peakon solution as follows:

$$
=\frac{\phi_{1}-\phi_{m} \operatorname{sech}^{2}\left(\omega|x-c t|+\tanh ^{-1} \sqrt{\left(\phi_{m}-\phi_{1}\right) / \phi_{m}}\right)}{\tanh ^{2}\left(\omega|x-c t|+\tanh ^{-1} \sqrt{\left(\phi_{m}-\phi_{1}\right) / \phi_{m}}\right)},
$$

where $\omega=(1 / 2) \sqrt{\Omega\left(\phi_{1}-\phi_{m}\right)}$. The profile of $(60)$ is shown in Figure $4(\mathrm{~m})$.

Substituting (58) into $d \phi / d \xi=y$ and integrating it along the homoclinic orbit yield the equation

$$
\int_{\phi_{m}}^{\phi} \frac{d s}{\left(\phi_{1}-s\right) \sqrt{s-\phi_{m}}}=\sqrt{\Omega}|\xi| .
$$

Completing the above integral, we can get a solitary wave solution as follows:

$$
u(x, t)=\phi_{m}-\left(\phi_{1}-\phi_{m}\right) \tanh ^{2}(\omega(x-c t)),
$$

where $\omega=(1 / 2) \sqrt{\Omega\left(\phi_{1}-\phi_{m}\right)}$. The profile of (62) is shown in Figure 4(n).

\subsection{Smooth Periodic Wave Solutions}

(i) For given $k>1$ and $h=0$ in Figure $1(\mathrm{~d})$, the level curve is shown in Figure 3(c). From Figure 3(c), we see that there is one periodic orbit enclosing the center point $\left(\phi_{1}, 0\right)$ of systems (9) and (10) and passing points $(0,0),\left(\phi_{M}, 0\right)$ when $(c, g) \in A_{4}$, where $\phi_{1}=-c$ and $\phi_{M}=-((k+1) / k) c$. Its expression is

$$
y= \pm \frac{\sqrt{k+1}}{k+1} \sqrt{\phi\left(\phi_{M}-\phi\right)}, \quad 0 \leq \phi \leq \phi_{M} .
$$

Substituting (63) into $d \phi / d \xi=y$ and integrating it along the periodic orbit yield the equation

$$
\int_{\phi}^{\phi_{M}} \frac{d s}{\sqrt{s\left(\phi_{M}-s\right)}}=\frac{\sqrt{k+1}}{k+1}|\xi| .
$$


Completing the above integral, we can get a smooth periodic wave solution as follows:

$$
u(x, t)=\phi_{M} \cos ^{2}(\omega(x-c t)),
$$

where $\omega=\sqrt{k+1} / 2(k+1)$. The profile of (65) is shown in Figure 4(o).

(ii) For given $k>1$ and $h=0$ in Figure $1(\mathrm{~g})$, the level curve is shown in Figure 3(d). From Figure 3(d), we see that there is one periodic orbit enclosing the center point $\left(\phi_{2}, 0\right)$ of systems (9) and (10) and passing points $(0,0),\left(\phi_{m}, 0\right)$ when $(c, g) \in A_{7}$, where $\phi_{2}=-c$ and $\phi_{m}=-((k+1) / k) c$. Its expression is

$$
y= \pm \frac{\sqrt{k+1}}{k+1} \sqrt{-\phi\left(\phi-\phi_{m}\right)}, \quad \phi_{m} \leq \phi \leq 0 .
$$

Substituting (66) into $d \phi / d \xi=y$ and integrating it along the periodic orbit yield the equation

$$
\int_{\phi_{m}}^{\phi} \frac{d s}{\sqrt{s\left(\phi_{m}-s\right)}}=\frac{\sqrt{k+1}}{k+1}|\xi| .
$$

Completing the above integral, we can get a smooth periodic wave solution as follows:

$$
u(x, t)=\phi_{m} \cos ^{2}(\omega(x-c t)),
$$

where $\omega=\sqrt{k+1} / 2(k+1)$. The profile of (68) is shown in Figure 4(p).

(iii) For given $k=-2$ and $h>0$ in Figure 2(f), the level curve is shown in Figure 3(j). From Figure 3(j), we see that there is one periodic orbit passing points $(0,0),\left(\phi_{m}, 0\right)$ when $(c, g) \in B_{6}$, where $\phi_{m}=-(1-\sqrt{1-2 h c}) / 2 h$. Its expression is

$$
y= \pm \sqrt{h} \sqrt{\left(\phi_{M}-\phi\right)(0-\phi)\left(\phi-\phi_{m}\right)}, \quad \phi_{m} \leq \phi \leq 0,
$$

where $\phi_{M}=(-1+\sqrt{1-2 h c}) / 2 h$.

Substituting (69) into $d \phi / d \xi=y$ and integrating it along the periodic orbit yield the equation

$$
\int_{\phi_{m}}^{\phi} \frac{d s}{\sqrt{\left(\phi_{M}-s\right)(0-s)\left(s-\phi_{m}\right)}}=\sqrt{h}|\xi| .
$$

Completing the above integral, we can get a smooth periodic wave solution as follows:

$$
u(x, t)=\phi_{m} c n^{2}(\omega(x-c t), k),
$$

where $\omega=(1 / 2) \sqrt{h\left(\phi_{M}-\phi_{m}\right)}$ and $k=\sqrt{\phi_{m} /\left(\phi_{m}-\phi_{M}\right)}$. The profile of (71) is shown in Figure 4(q).

(iv) For given $k=-2$ and $h<0$ in Figure 2(i), the level curve is shown in Figure 3(k). From Figure 3(k), we see that there is one periodic orbit passing points $(0,0),\left(\phi_{M}, 0\right)$ when $(c, g) \in B_{9}$, where $\phi_{M}=-(1-\sqrt{1-2 h c}) / 2 h$. Its expression is

$$
y= \pm \sqrt{-h} \sqrt{\left(\phi_{M}-\phi\right)(\phi-0)\left(\phi-\phi_{m}\right)}, \quad 0 \leq \phi \leq \phi_{M},
$$

where $\phi_{m}=-(1+\sqrt{1-2 h c}) / 2 h$.
Substituting (72) into $d \phi / d \xi=y$ and integrating it along the periodic orbit yield the equation

$$
\int_{\phi}^{\phi_{M}} \frac{d s}{\sqrt{\left(\phi_{M}-s\right)(s-0)\left(s-\phi_{m}\right)}}=\sqrt{-h}|\xi| .
$$

Completing the above integral, we can get a smooth periodic wave solution as follows:

$$
u(x, t)=\phi_{M} c n^{2}(\omega(x-c t), k),
$$

where $\omega=(1 / 2) \sqrt{h\left(\phi_{m}-\phi_{M}\right)}$ and $k=\sqrt{\phi_{m} /\left(\phi_{m}-\phi_{M}\right)}$. The profile of (74) is shown in Figure 4(r).

\section{Conclusion}

In this paper, using the bifurcation theory and the method of phase portraits analysis, we investigated a modification of the $K(2,2)$ equation $(6)$ and obtained some peakon, solitary, and smooth periodic wave solutions. We also show that when $(c, g) \in B_{3}$ and $(c, g) \in B_{12}$, the peakon and the solitary wave coexist in (6). We can say that we obtained some new results of (6) in this paper.

\section{Conflict of Interests}

The authors declare that there is no conflict of interests regarding the publication of this paper.

\section{Acknowledgments}

The authors would like to thank the reviewers very much for their useful comments and helpful suggestions. This work is supported by the Natural Science Foundation of Yunnan Province, China (no. 2013FZ117), and the National Natural Science Foundation of China (no. 11161020).

\section{References}

[1] P. Rosenau and J. M. Hyman, "Compactons: solitons with finite wavelength," Physical Review Letters, vol. 70, no. 5, pp. 564-567, 1993.

[2] P. Rosenau, "On nonanalytic solitary waves formed by a nonlinear dispersion," Physics Letters A, vol. 230, no. 5-6, pp. 305-318, 1997.

[3] P. Rosenau, "Nonlinear dispersion and compact structures," Physical Review Letters, vol. 73, no. 13, pp. 1737-1741, 1994.

[4] P. Rosenau, "On a class of nonlinear dispersive-dissipative interactions," Physica D, vol. 123, no. 1-4, pp. 525-546, 1998.

[5] A.-M. Wazwaz, "General compactons solutions for the focusing branch of the nonlinear dispersive $K(n, n)$ equations in higherdimensional spaces," Applied Mathematics and Computation, vol. 133, no. 2-3, pp. 213-227, 2002.

[6] A.-M. Wazwaz, "General solutions with solitary patterns for the defocusing branch of the nonlinear dispersive $K(n, n)$ equations in higher dimensional spaces," Applied Mathematics and Computation, vol. 133, no. 2-3, pp. 229-244, 2002.

[7] A.-M. Wazwaz, "A reliable treatment of the physical structure for the nonlinear equation $K(m, n)$," Applied Mathematics and Computation, vol. 163, no. 3, pp. 1081-1095, 2005. 
[8] L. X. Tian and J. L. Yin, "Shock-peakon and shock-compacton solutions for $K(p, q)$ equation by variational iteration method," Journal of Computational and Applied Mathematics, vol. 207, no. 1, pp. 46-52, 2007.

[9] A. Biswas, "1-soliton solution of the $K(m, n)$ equation with generalized evolution and time-dependent damping and dispersion," Computers and Mathematics with Applications, vol. 59, no. 8, pp. 2536-2540, 2010.

[10] A. Biswas, "1-soliton solution of the $K(m, n)$ equation with generalized evolution," Physics Letters A, vol. 372, no. 25, pp. 4601-4602, 2008.

[11] G. Ebadi and A. Biswas, "The $G^{\prime} / G$ method and topological soliton solution of the $K(m, n)$ equation," Communications in Nonlinear Science and Numerical Simulation, vol. 16, no. 6, pp. 2377-2382, 2011.

[12] M. Incay, E. Ulutasb, E. Cavlaka, and A. Biswas, "Singular 1-soliton solution of the $K(m, n)$ equation with generalized evolutions and its subsidiaries," Acta Physica Polonica B, vol. 44, pp. 1825-1836, 2013.

[13] D. M. Ambrose and J. D. Wright, "Travelingwaves and weak solutions for an equation with degenerate dispersion," http://www.drexel.edu/math/about/ /media/Files/math/ annual-report/\%202009-2010-annual-report.ashx.

[14] J. B. Li and H. H. Dai, On the Study of Singular Nonlinear Traveling Wave Equation: Dynamical System Approach, Science Press, Bejing, China, 2007.

[15] J. B. Li and Z. R. Liu, "Smooth and non-smooth traveling waves in a nonlinearly dispersive equation," Applied Mathematical Modelling, vol. 25, no. 1, pp. 41-56, 2000.

[16] M. Song, Z. R. Liu, E. Zerrad, and A. Biswas, "Singular solitons and bifurcation analysis of quadratic nonlinear Klein-Gordon equation," Applied Mathematics \& Information Sciences, vol. 7, pp. 1333-1340, 2013.

[17] B. He, "Bifurcations and exact bounded travelling wave solutions for a partial differential equation," Nonlinear Analysis: Real World Applications, vol. 11, no. 1, pp. 364-371, 2010. 


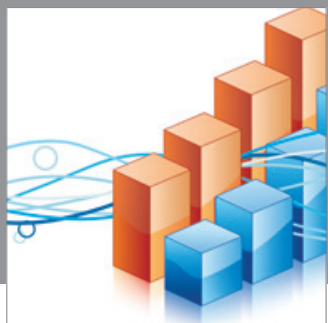

Advances in

Operations Research

mansans

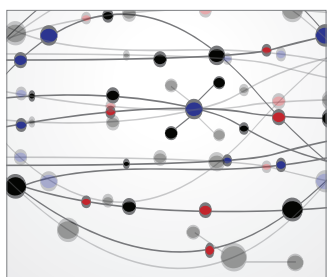

The Scientific World Journal
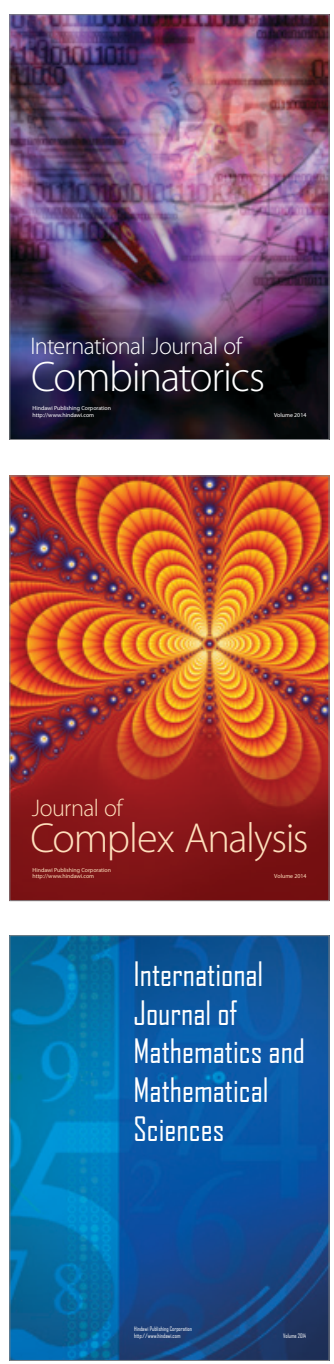
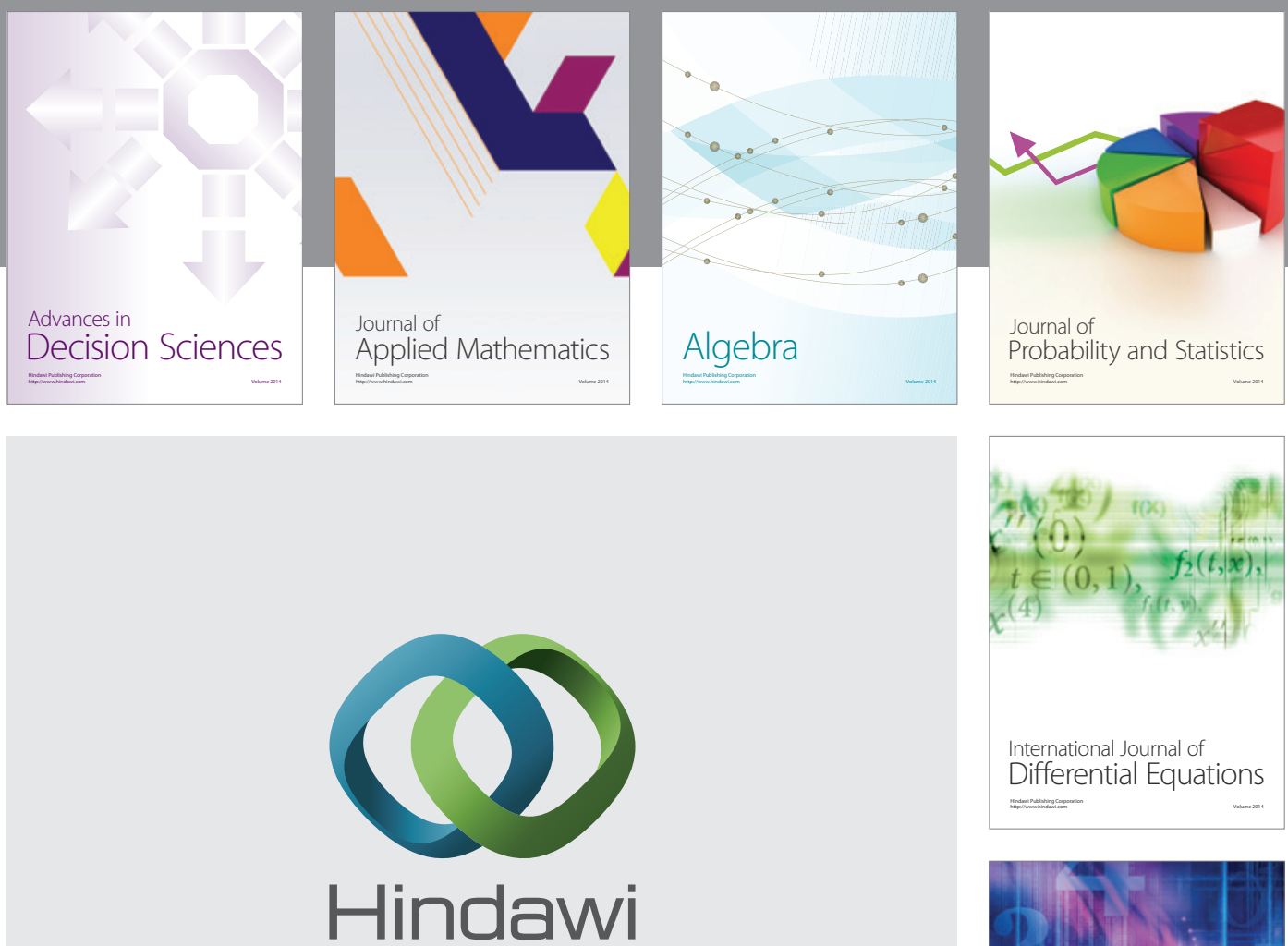

Submit your manuscripts at http://www.hindawi.com
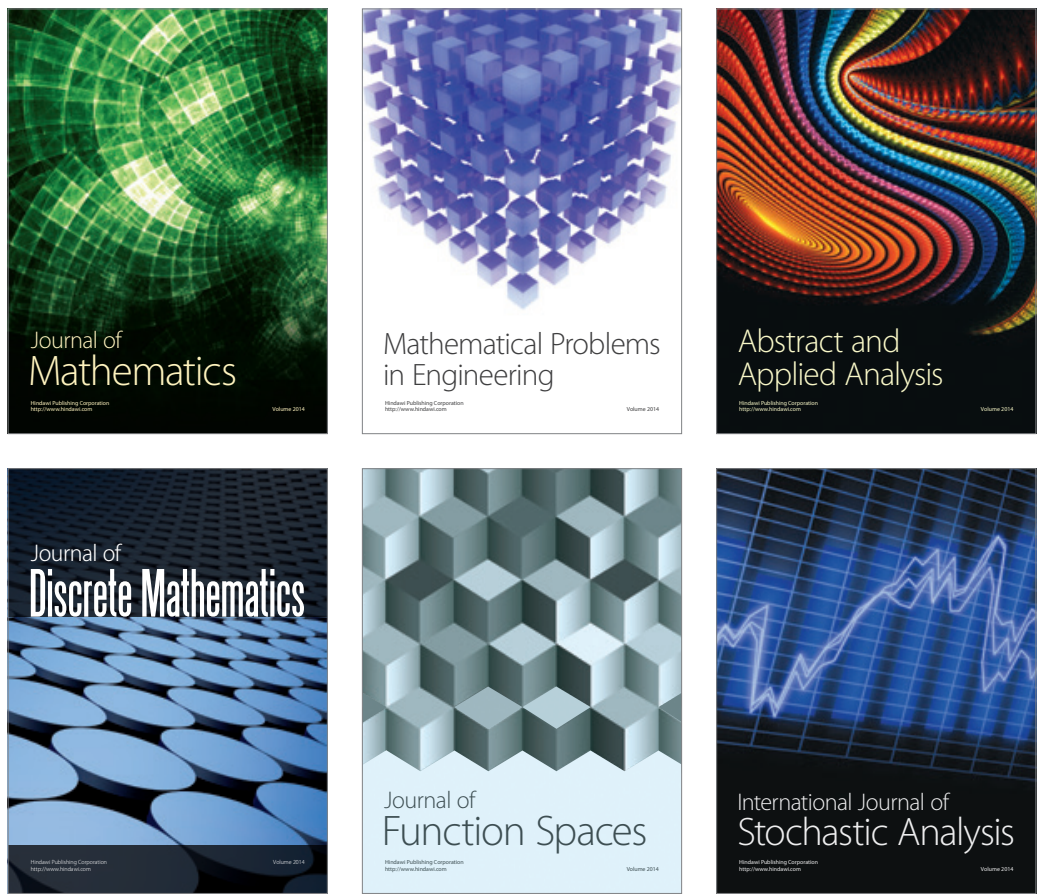

Journal of

Function Spaces

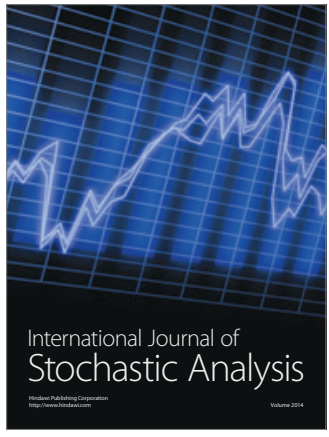

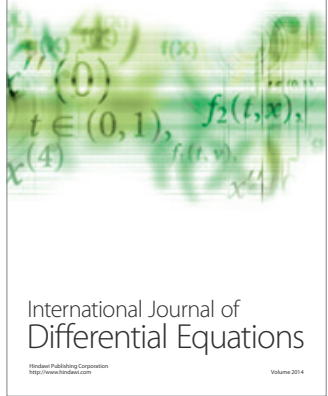
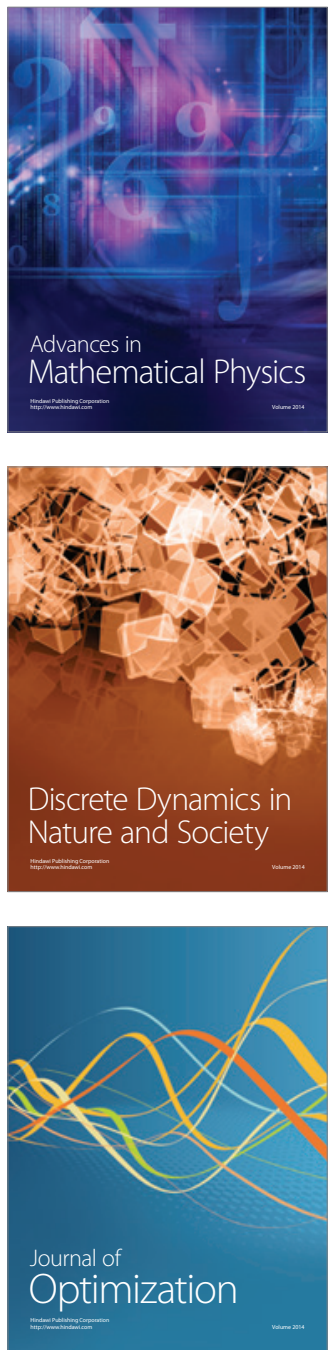